\title{
Cataract surgery and subtype in a defined, older population: the SEECAT Project
}

\author{
A Lewis, N Congdon, B Munoz, H Bowie, H Lai, P Chen, S K West
}

Br J Ophthalmol 2004;88:1512-1517. doi: 10.1136/bjo.2004.045484

Aim: To describe the distribution of cataract subtypes present before surgery among a defined population of older, bilaterally pseudophakic individuals.

Methods: This was a cohort study of bilaterally pseudophakic individuals participating in the Salisbury Eye Evaluation (SEE), and their locally resident siblings. Subjects underwent slit lamp and retroillumination photography and grading using the Wilmer Cataract Grading System. For all individuals determined to be bilaterally pseudophakic, an attempt was made to determine for each eye the type(s) of cataract present before surgery, based on previous SEE photographs (for SEE participants) and/or medical records obtained from the operating ophthalmologist (for both SEE participants and their siblings).

Results: The mean age of 223 participants providing data in this study was 78.7 (SD 5.2) years, $19.3 \%$ of subjects were black and $60.1 \%$ female. The most common surgically removed cataract subtype in this population was pure nuclear $(43.5 \%)$, followed by nuclear combined with posterior subcapsular cataract (PSC) $(20.6 \%)$, and nuclear combined with cortical (13.9\%); less common types were pure cortical $(4.9 \%)$, pure PSC $(4.5 \%)$, and PSC combined with cortical $(2.7 \%)$. Factors such as sex and source of lens data (study photograph versus clinical record) did not significantly affect the distribution of lens opacity types, while PSC was significantly $(p=0.01)$ more common among younger people and nuclear cataract was significantly $(p=0.001)$ more common among white compared to black people.

Conclusion: Epidemiological studies have suggested that the different subtypes of cataract are associated with different risk factors. As studies begin to identify new prevention strategies for cataract, it would appear likely that different strategies will be efficacious against different types of cataract. In this setting, it will be helpful to know which cataract types are most frequently associated with surgery. Among this older, majority white population, nuclear cataract showed a clear predominance among individuals having undergone surgery in both eyes. This may be contrasted with both clinic and population based studies of younger people, which have generally found PSC cataract to predominate.

\footnotetext{
A
} ge related cataract is the leading cause of blindness in the world, ${ }^{1}$ and the most common cause of visual disability among older Americans. ${ }^{2}$ It is a treatable cause of decreased vision, accounting for visual impairment in more than $25 \%$ of women and $12 \%$ of men aged 75 years and older in a Beaver Dam (WI) population with ready access to cataract surgical services. ${ }^{3}$ Unoperated cataract is a particularly prevalent cause of blindness among AfricanAmerican people. ${ }^{4}$

With changing indications for cataract surgery, cataracts are now removed more frequently, and earlier in a patient's life than in previous decades. ${ }^{5}$ Klein et al ${ }^{6}$ suggest that age related cataract is not uncommon as early as the fifth decade of life, with $11.5 \%$ of people aged $43-54$ years having some evidence of cortical opacity. The medical economic impact of cataract surgery is significant, as cataract care consumes $60 \%$ of the Medicare budget for vision. ${ }^{7}$ Payments for evaluation and surgical treatment of cataract consume two thirds of the total Medicare budget related to vision care. ${ }^{7}$

The demographic and other risk factors associated with lens opacities undergoing surgical removal have been addressed in several publications. ${ }^{38-12}$ However, the literature describing the specific cataract subtypes (nuclear, cortical, or posterior subcapsular cataract (PSC)) requiring removal in a defined population is relatively sparse. Adamsons et al report that PSC was present in nearly two thirds of lenses undergoing cataract surgery in a clinic based series, ${ }^{13}$ while Klein and colleagues ${ }^{3}$ similarly report PSC as the most important lens opacity in predicting incident cataract surgery in a population based study. The apparent high prevalence of PSC among cataracts undergoing extraction is in clear distinction to its prevalence in the population at large, where PSC represents the least common subtype in nearly all population based studies. ${ }^{14}$

We recently carried out a study of the genetics of age related cataract in an older population drawn from participants in the Salisbury Eye Evaluation (SEE) project $^{15}$ in Salisbury, Maryland, and their siblings. Because of the age of the population involved, bilateral pseudophakia was quite common; in order to avoid loss of power, an effort was made to identify the type of cataract present in each eye for all such individuals, through use of previous photographs in the SEE project and/or records provided by the operative surgeon. Based on these data, we now report on the cataract subtype present before surgery in a well defined older population and their siblings.

\section{METHODS}

Between 1993 and 1995, the Salisbury Eye Evaluation (SEE) Project enrolled a random sample of 2520 residents of the Salisbury, Maryland area who were identified by Medicare records as 65-84 years old. Of the 1504 participants in the third round of the project, those with one or more siblings residing within 100 miles of Baltimore or Salisbury were invited to participate as "probands" in the SEE Cataract Genetics Study (SEECAT). After giving informed consent, all such probands and their siblings were examined at a central site, where a full ocular examination including dilatation of

Abbreviations: PSC, posterior subcapsular cataract; SEE, Salisbury Eye Evaluation; SEECAT, SEE Cataract Genetics Study 


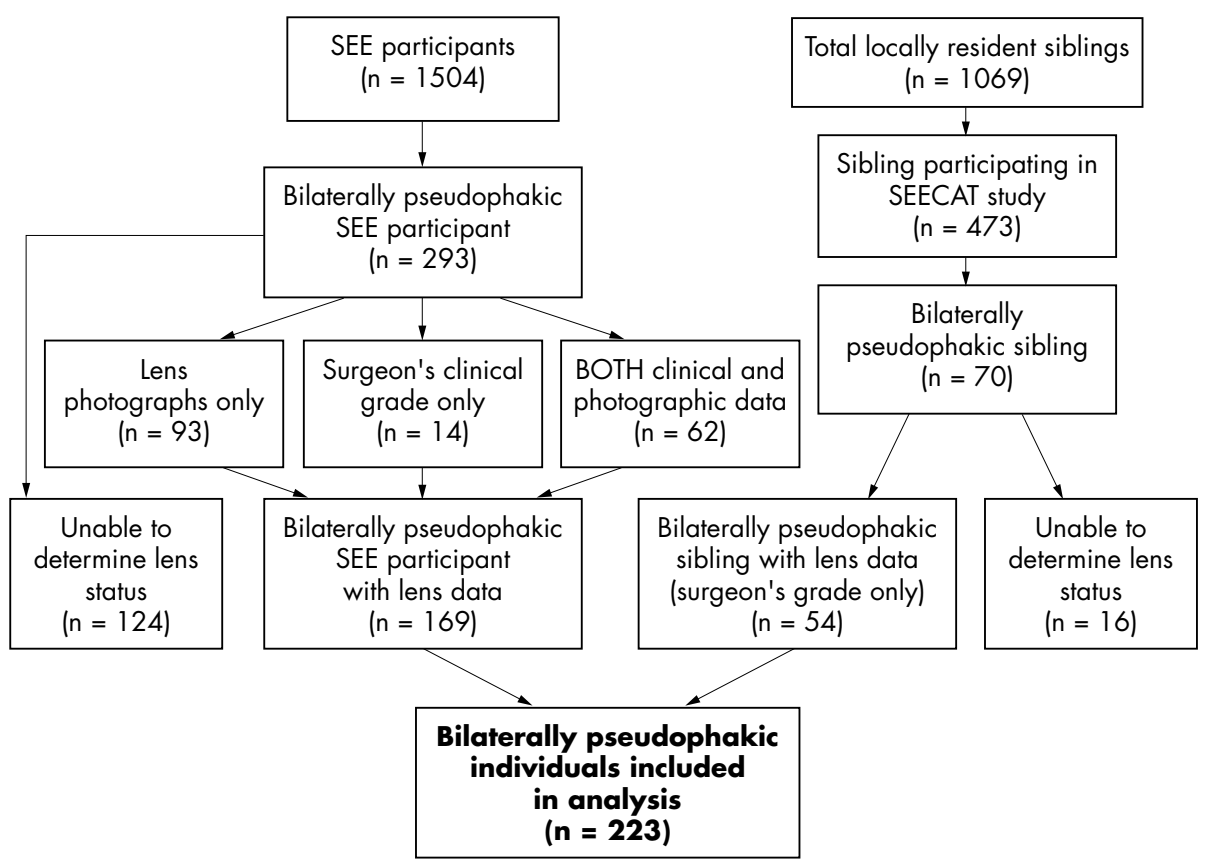

Figure 1 Subjects in the Salisbury Eye Evaluation (SEE) project and their locally resident siblings participating in a study of cataract subtype present before surgery in bilaterally pseudophakic individuals. the pupil was carried out by an optometrist (HB), who determined if cataract surgery had occurred.

Photographs from previous rounds of the SEE project (for "probands") and/or records of the preoperative clinical evaluation by the operating ophthalmologist (for both probands and siblings) were utilised in an attempt to determine the cataract subtype(s) present before surgery for one or both eyes in all subjects deemed to be bilaterally pseudophakic/aphakic. The Wilmer Cataract Grading System used in this study has been elaborated in detail elsewhere. ${ }^{16}$ Briefly, after dilatation of the pupil, standardised slit lamp photographs (Topcon SL-5D) were taken to assess nuclear change, and retroillumination photographs (Neitz camera) were taken to assess cortical and posterior subcapsular opacities. ${ }^{16}{ }^{17}$ Slit lamp photographs were carried out with a $0.1 \mathrm{~mm}$ slit beam, at a height of $8-9 \mathrm{~mm}$, set at an angle of $30^{\circ}$. A pair of retroillumination photographs, one focused on the anterior aspect of the lens and the other on its posterior aspect, were made for each eye. Nuclear cataracts were graded in decimal increments from 0.1 to 4.0 with reference to four standard photographs. A reference circle divided into 16 wedges was used to grade cortical cataracts from 0 to 16 out of 16, according to the proportion of the lens involved by cortical opacity. PSC was graded as present or absent. ${ }^{16}{ }^{17} \mathrm{~A}$ subject was defined as "affected" by a particular cataract subtype if either eye:

- was affected by that subtype on a previously graded SEE photograph (nuclear grade $\geqslant 2$ out of 4 , cortical cataract grade $\geqslant 4$ out of 16 , PSC cataract present)

or

- had cataract of that subtype graded by the operative surgeon as $2+$ or greater (on a scale of 4 ), or characterised as "dense," "significant," or similar terminology in a clinical chart.

Clinician grades might often come several years after the most recent previous study photograph, and so frequently provided new information. All cataract subtype designations

Table 1 Demographic characteristics of SEE participants and their locally resident siblings. $\chi^{2}$ tests compared the group of bilateral pseudophakics with lens data to the population base of probands or siblings

\begin{tabular}{|c|c|c|c|}
\hline & $\begin{array}{l}\text { Mean age (SD) } \\
\text { (years)* }\end{array}$ & $\begin{array}{l}\text { Race } \\
\text { (\% black }) \dagger\end{array}$ & $\begin{array}{l}\text { Sex } \\
(\% \text { female }) \ddagger\end{array}$ \\
\hline \multicolumn{4}{|l|}{ SEE participants } \\
\hline All SEE participants $(n=1504)$ & $78.2(4.7)$ & 24.5 & 58.9 \\
\hline Bilaterally pseudophakic SEE participants $(n=293$ ) & $79.9(4.9)$ & 16.7 & 65.5 \\
\hline $\begin{array}{l}\text { Bilaterally pseudophakic SEE participants with lens data } \\
(n=169)\end{array}$ & $79.2(4.8)$ & 18.9 & 62.7 \\
\hline \multicolumn{4}{|l|}{ Siblings } \\
\hline Locally resident siblings $(n=1069)$ & $71.1(9.7)$ & 34.0 & 60.0 \\
\hline Siblings participating in SEECAT study $(n=473)$ & $70.2(7.7)$ & 26.8 & 59.0 \\
\hline Bilaterally pseudophakic siblings $(n=70)$ & $76.2(6.0)$ & 15.7 & 60.0 \\
\hline Bilaterally pseudophakic siblings with lens data $(n=54)$ & 75.9 (6.8) & 18.5 & 51.8 \\
\hline
\end{tabular}

*Age did not differ significantly for bilaterally pseudophakic participants with and without lens data among SEE participants $(p=N S)$ or siblings $(p=N S)$.

†Race did not differ significantly for bilaterally pseudophakic participants with and without lens data among SEE participants $(P=N S)$ or siblings $(p=N S)$.

¥Sex did not differ significantly for bilaterally pseudophakic participants with and without lens data among SEE participants $(p=N S)$ or siblings $(p=N S)$. 


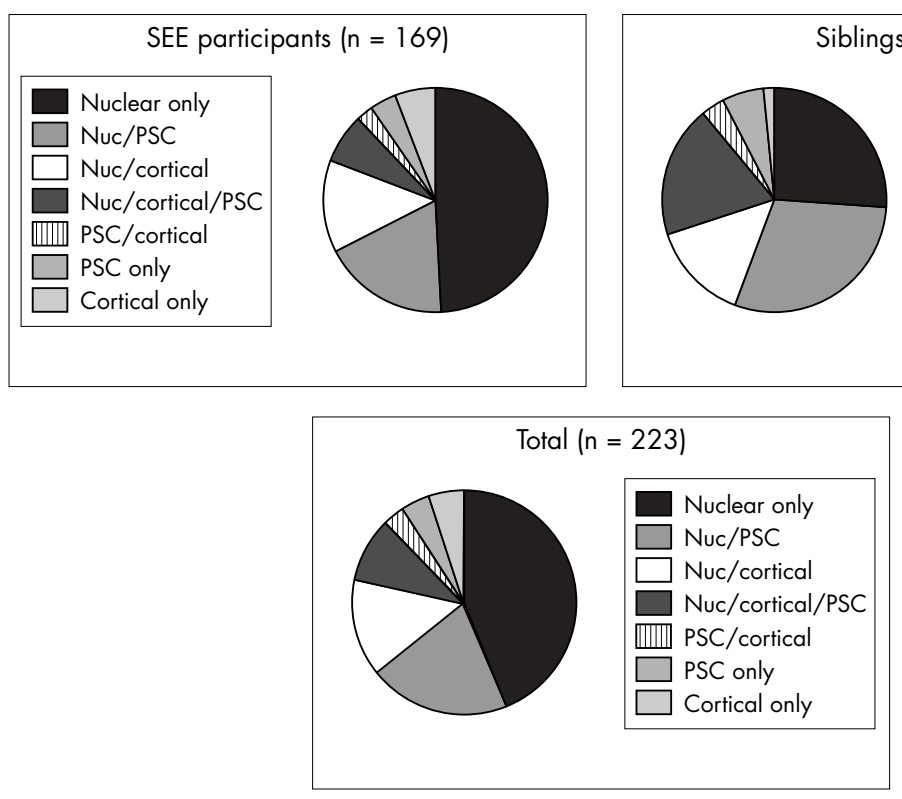

Figure 2 Cataract subtype distribution among SEE participants, siblings, and all participants in a study of cataract subtype and cataract surgery. were assigned by a single reviewing ophthalmologist (NGC). Presence of cataract subtypes was not mutually exclusive. Individuals affected in one eye by one subtype of cataract and in the other eye by another would be identified as affected by both subtypes. In those eyes where a determination of pseudophakia or aphakia was not consistent between the clinical examination and photographs, adjudication was carried out by the principal investigator (NGC) based on a review of photographs and clinical records. The protocol described above was reviewed in full by the joint committee on clinical investigation, the institutional review board for the Johns Hopkins University School of Medicine.

\section{Statistical methods}

Stata statistical software was used in the analysis of these data. $^{18}$ Additionally, the GENMOD procedure in SAS (SAS Systems, Cary, NC, USA) was used to fit a GEE using maximum likelihood estimation of parameters to take into account the correlation in lens grades between siblings. Since the various cataract types were constrained to sum to $100 \%$, and were thus not independent of one another, comparisons between cataract subtype distributions for race, sex, age, etc, were made only for one subtype at a time, treated as a "reference" type according to the binomial distribution. All analyses were carried out by a person, reflecting the presence of cataract type or pseudophakia/aphakia in either eye.

\section{RESULTS}

A total of 363 subjects (293 SEE participants and 70 siblings) were bilaterally pseudophakic/aphakic (fig 1). Among these subjects, previous photographs and/or preoperative clinical records were available for 223 people $(61.4 \%)$, who are included in all further analyses. This number is composed of 65 SEE participants with a sibling participating in SEECAT, 104 SEE participants without a sibling participating in SEECAT, and 54 siblings. There were no statistically significant differences in race, age, or sex between the group of SEE participants with siblings $(n=65)$ and the group without siblings $(\mathrm{n}=104)$; these two groups are combined in all further analyses.

As expected, among both SEE participants and siblings, bilaterally pseudophakic subjects were somewhat older and were less likely to be black compared to the group as a whole (table 1). The race, age, and sex characteristics of bilaterally pseudophakic subjects did not differ significantly for those with and without available lens data for either SEE participants or siblings (table 1 ).

The distribution of age $(p=0.54)$, sex $(p=0.16)$, and race $(\mathrm{p}=0.95)$ among bilaterally pseudophakic SEE participants with lens data $(n=169)$ did not differ from the group of bilaterally pseudophakic siblings with lens data $(n=54)$.

The distribution of cataract subtype was similar between SEE participants and siblings, with nuclear cataract predominating in both groups (fig 2). When the two groups were combined, pure nuclear cataract was the most common subtype $(43.5 \%)$, followed by nuclear in combination with PSC $(20.6 \%)$, and nuclear combined with cortical opacity $(13.9 \%)$. Cataracts composed of all three subtypes (PSC, cortical, and nuclear) were found among 9.9\% of the entire group. The least common cataract subtype in this study population was posterior subcapsular combined with cortical $(2.7 \%)$. The proportion of subjects with "any" significant nuclear cataract noted in the medical record or in a lens photograph in either eye was $87.5 \%$ (95\% CI 83.6 to 92.2 ), while $37.9 \%$ had "any" PSC and 30.8\% had "any" cortical opacity.

Nuclear cataract predominated for both male and female subjects, and the proportion of nuclear cataract did not differ by sex when adjusting for between sibling correlations $(p=0.12)$. Any PSC was significantly $(p=0.01$, adjusted for between sibling correlations) more common among subjects younger than the mean age for the group (78.7 years) as compared to those at or above the mean age. Any nuclear cataract was significantly $(p=0.001$, adjusted for between sibling correlations) more common among white persons compared to black people (fig 3 ).

Although the small numbers involved precluded formal statistical testing, the source of lens data, whether from previous SEE photographs or the clinicians' records, did not appear to affect the reported cataract subtype significantly. Pure nuclear cataract predominated among subjects with only photographs $(n=93)$, only clinician grades $(n=14)$, and those with both photographs and clinicians' grades $(n=62)$ (fig 4$)$. Note that, as only SEE participants $(n=169)$ had previous SEE photographs, only their data could be included in this analysis.

Nuclear cataract predominated among both first eye $(n=139)$ and second eye $(n=137)$ surgeries (fig 5$)$. Note 

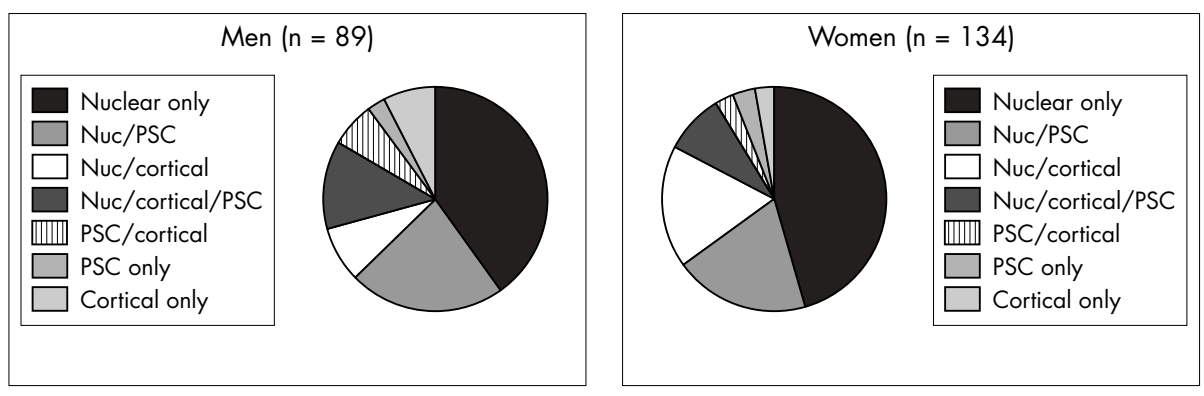

Figure 3 Sex, race, age, and cataract subtype distribution in a study of cataract subtype and cataract surgery.
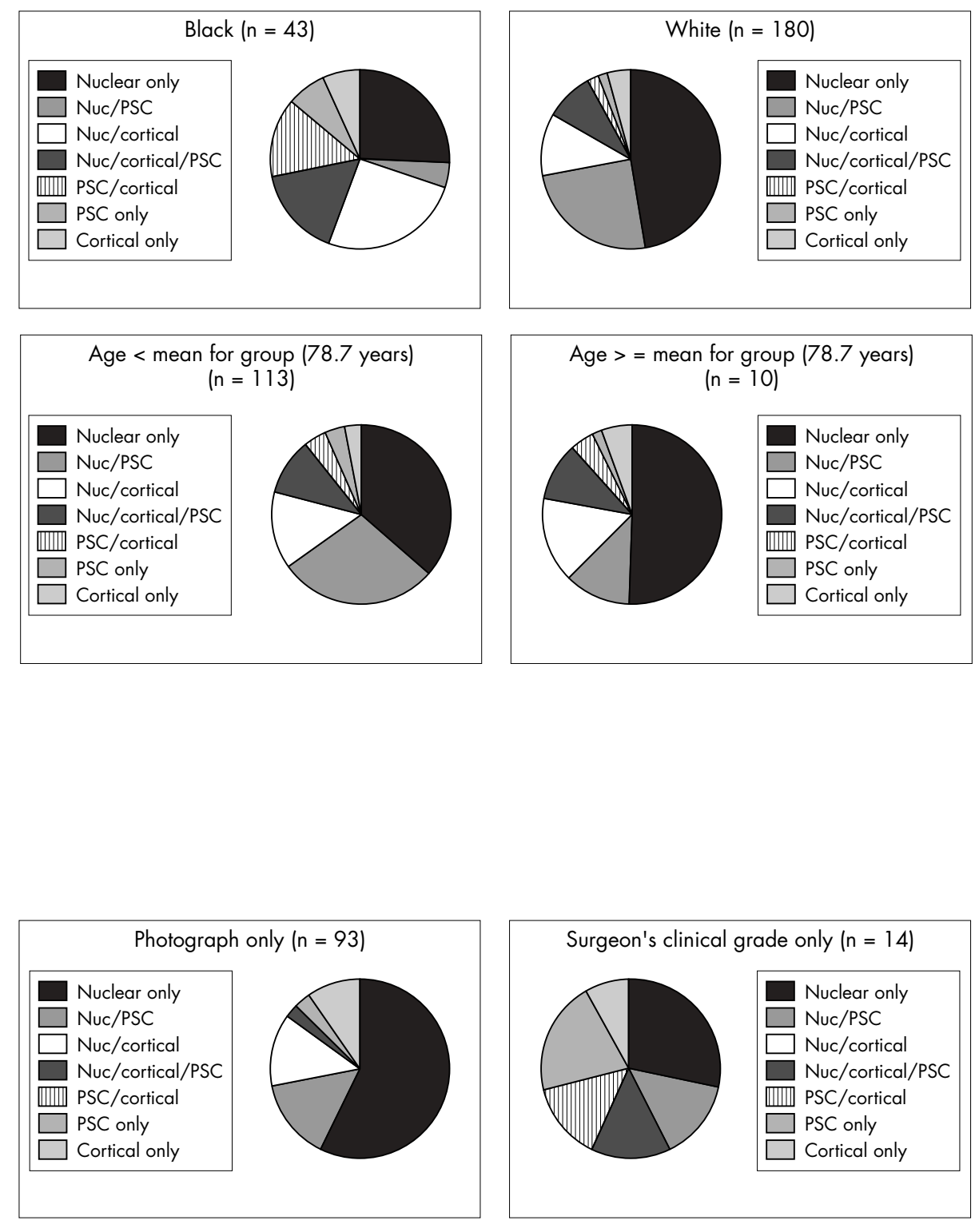

Figure 4 Type of lens data available and cataract subtype distribution among SEE participants in a study of cataract subtype and cataract surgery.
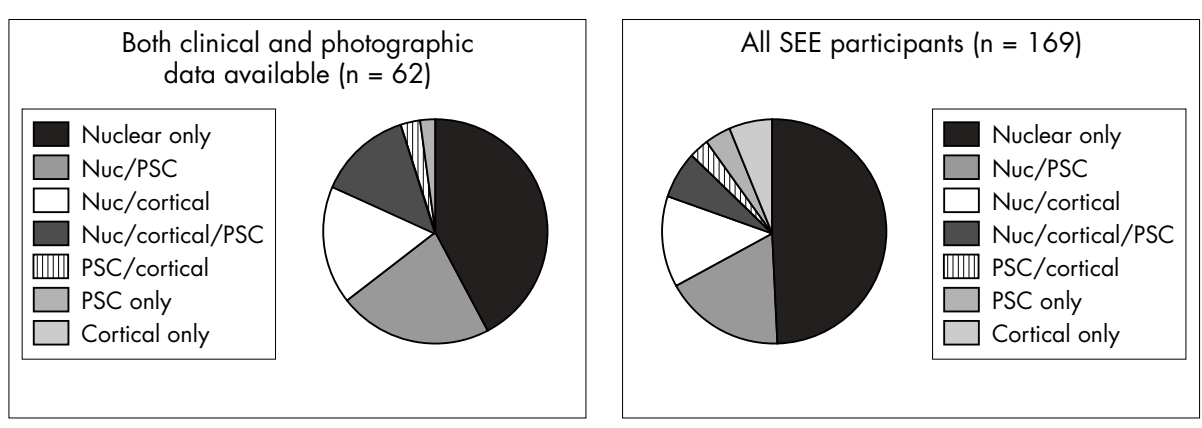

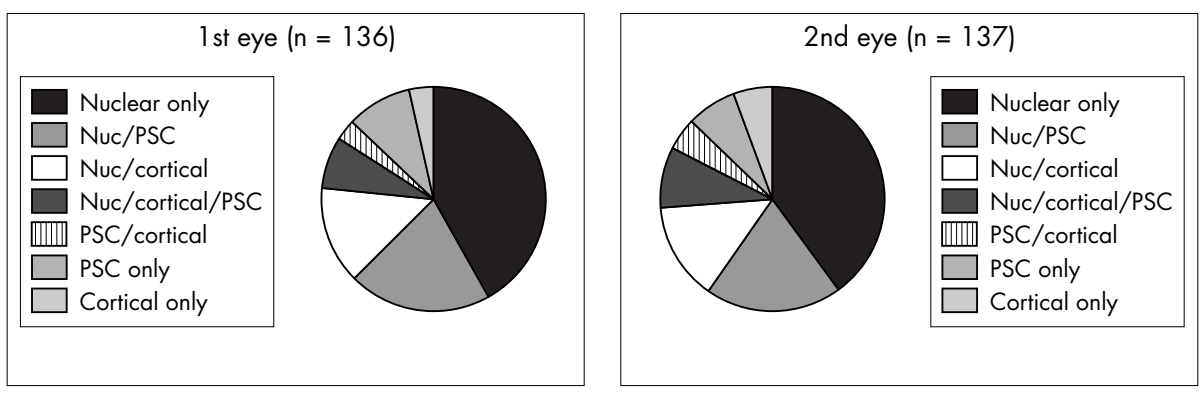

Figure 5 Order of surgery (first eye versus second eye) and the distribution of cataract subtypes in a study of cataract subtype and cataract surgery.

that only individuals for whom the dates of surgery for both eyes were known could be included in this analysis.

\section{DISCUSSION}

In this well defined older population of bilaterally pseudophakic people, nuclear cataract clearly predominated as the subtype present before surgery, with pure nuclear and nuclear mixed with other subtypes accounting for nearly $90 \%$ of opacities. This pattern was observed regardless of whether the cataract subtype was identified by photographic grading, clinician assessment or both. There can be no doubt of the importance of obtaining information on cataract surgery among older populations such as this: it is estimated that some $62 \%$ of cataract surgery is performed in the United States on people aged 75 years and above. ${ }^{19}$

In contrast with our results, PSC has been identified as the type of cataract associated with the greatest risk for subsequent cataract surgery in population based studies from Blue Mountains in Australia ${ }^{20}$ and Beaver Dam in the United States. ${ }^{3}$ Additionally, clinic based studies have also reported a predominance of PSC in surgical series. ${ }^{13} 21$

One possible reason for this apparent disparity may relate to age differences in the populations studied. Several surgical series $^{1321}$ and population studies ${ }^{3}$ have reported that people developing or undergoing surgery for PSC cataract are on average significantly younger than those with nuclear cataract. Our own data show that PSC was significantly more common before cataract surgery among younger people compared to older participants. The younger group of siblings also appeared to have somewhat more PSC than the older group of SEE participants. The mean age in our study population was in the late 70s, a decade older, for example, than the mean for Adamsons et al's subjects (67.5 years). ${ }^{13}$ The mean age at the time of surgery for our subjects was only 70.8 (SD 7.8) years, still several years older than the group reported by Adamsons et al. Although our subjects on average underwent surgery several years before the time when they presented to Round 3 of the SEE study, a similar time lag would also be present in other surgical incidence studies such as those from Blue Mountains ${ }^{20}$ and Beaver Dam, both of which reported on younger populations than ours. ${ }^{3}$

The possibility of bias, introduced by our inability to find older medical records pertaining to surgeries performed earlier in life, cannot be excluded, though information was obtained for some $62 \%$ of subjects. For example, nearly two thirds of the remaining $38 \%$ of subjects would have had to have pure PSC to have made PSC the most common form of cataract among all subjects (fig 2). The observed rate of pure PSC was only $4.5 \%$ (95\% CI 1.8 to 7.2 ) among subjects with lens information.

Alternatively, increased mortality associated with PSC might explain our failure to detect PSC or a history of cataract surgery for PSC in the older population we examined. In fact, all subtypes of cataract, not only PSC, have been associated with increased mortality, perhaps as a surgery. ${ }^{22-24}$ It has recently been suggested that increased mortality associated with cataract may actually be related to cataract risk factors such as diabetes, smoking, and cardiovascular disease, rather than cataract itself. ${ }^{24}$

Despite differences in subject age and methodology between the current study and those previously published it is, none the less, interesting to examine more closely the question of whether PSC cataract predominates in surgical studies. Adamsons et al, for example, conclude that "(PSC cataracts) cause more significant visual disability than do other types of cataracts." However, among 158 subjects undergoing a total of 198 cataract extractions in a general ophthalmology practice, Adamsons and colleagues report that preoperative clinical examination disclosed significant nuclear opacity in $65 \%$ of patients, as opposed to PSC in $61 \%{ }^{13}$ Summarising the Beaver Dam data on incident cataract surgery, Klein et al state that "Many factors are associated with cataract surgery, but the prior presence of posterior subcapsular cataract is the most important lens opacity." However, the age adjusted incidence of cataract surgery among 360 people with any nuclear cataract at baseline in the Beaver Dam study was $32.0 \%$, compared to $28.0 \%$ for 112 people with any PSC. While the increased odds of undergoing incident cataract surgery associated with the presence of PSC (OR 15.51, 95\% CI 8.27 to 29.09) was higher than for nuclear cataract (OR 4.0, 95\% CI 2.40 to 6.66), the much higher prevalence of nuclear cataract appears to result in more people in the Beaver Dam population having undergone cataract extraction in association with nuclear as opposed to PSC opacity. ${ }^{3}$

Limitations of the current study must be clearly acknowledged. In the first place, both the SEE and SEECAT studies are relatively small ones, including 1500 and fewer than 800 subjects respectively. However, the advanced age of the study population guaranteed that the number of people undergoing cataract surgery was relatively high. For example, the total number of pseudophakic subjects for whom we were able to obtain lens information $(n=223)$ exceeds the number of incident cataract surgeries reported in the Blue Mountains Study $(\mathrm{n}=126)^{20}$ and approximately equals that for Beaver $\operatorname{Dam}(\mathrm{n}=226){ }^{3}$

Secondly, the current report does not represent a truly population based sample. In order to participate, subjects had to be bilaterally pseudophakic, and have available preoperative records and/or old study photographs. Participants recruited from the parent SEE project were additionally subject to the survivor effect inherent in having lived to take part in the most recent round of a decade long study. Unlike the Blue Mountains and Beaver Dam studies, the current report does not describe all people in the population undergoing cataract surgery. However, it was possible to demonstrate demographic similarities between study participants and all subjects taking part in the population based SEE project, and all living siblings of SEE subjects. result of higher rates of accidents or complications from 
Furthermore, the current report provides unique data on cataract surgery in an older population, known to consume the large majority of cataract surgical resources, and also includes a substantial number of black people, not included in previous cataract surgical studies. ${ }^{3}{ }^{13} 2021$

Finally, there are inevitable limitations in our attempts to reconstruct lens opacities existing at the time of surgery from a combination of previous study photographs and clinician reports. Clinicians were not standardised in their reporting, and may have introduced substantial error into the reported cataract subtype distribution. Specifically, there is likely to be under-reporting by clinicians of cortical opacity as a subtype. ${ }^{4}$ Balanced against this concern is the fact that substantial time may have elapsed between the time of study photographs and cataract extraction. Introducing clinician reports may have allowed us to capture incident changes in the lens which would otherwise have been lost, especially for PSC, which may progress rapidly.

This study of cataract subtype in a well defined, older population undergoing bilateral cataract extraction highlights the importance of nuclear cataract as a cause of visual impairment. While PSC appears to predominate in cataract surgical series including younger people, nuclear cataract assumes increasing importance in older groups. Our results suggest that nuclear cataract may convey a greater lifetime risk of cataract surgery. Given the ageing of the American population, and the fact that the very oldest segments of the population already consume the bulk of cataract surgical services, it is clear that nuclear cataract is a critical target for cataract prevention efforts.

\section{Authors' affiliations}

A Lewis, N Congdon, B Munoz, H Bowie, H Lai, P Chen, S K West, The Dana Center for Preventive Ophthalmology, Johns Hopkins University Schools of Medicine and Public Health, Baltimore, MD, USA

This work was supported by grants from the National Institute of Aging (R-01 16294), the National Eye Institute (K-23 EY00388) and Research to Prevent Blindness to Drs Congdon and West.

The authors have no financial interest in the material presented here.

Correspondence to: Dr Nathan Congdon, Wilmer 120, 600 N Wolfe Street, Baltimore, MD 21287, USA; ncongdon@jhmi.edu

Accepted for publication 4 May 2004

\section{REFERENCES}

1 Thylefors B, Negrel AD, Pararajasegaram R, et al. Global data on blindness. Bull World Health Organ 1995;73:115-21.

2 Eye Disease Prevalence Research Group. Causes and prevalence of visual impairment among adults in the United States. Arch Ophthalmol (in press).

3 Klein BEK, Klein R, Moss SE. Incident Cataract Surgery. Ophthalmology 1997; 104:573-80.

4 Munoz B, West SK, Rubin GS, et al. Causes of blindness and visual impairment in a population of older Americans: the Salisbury Eye Evaluation Study. Arch Ophthalmol 2000;118:819-25.

5 Superstein R. Indications for cataract surgery. (Review) Curr Opin Ophthalmol, 2001;12:58-62.

6 Klein BEK, Klein R, Lee KE. Incidence of age-related cataract over a 10-year interval. Ophthalmology 2002;109:2052-7.

7 Ellwein LB, Urato CJ. Use of eye care and associated charges among the Medicare population: 1991-1998. Arch Ophthalmol 2002;120:804-11.

8 Cruickshanks KJ, Klein BEK, Klein R. Ultraviolet light exposure and lens opacities: The Beaver Dam Eye Study. Am J Public Health 1992;82:1658-62.

9 Klein BEK, Klein R, Lee KE. Incident cataract after five-year interval and lifestyle factors: The Beaver Dam Eye Study. Ophthalmic Epidemiol 1999;6:247-55.

10 Klein BEK, Klein R, Lee KE. Diabetes, cardiovascular disease, selected cardiovascular disease risk factors, and the 5-year incidence of age-related cataract and progression of lens opacities: the Beaver Dam Eye Study. Am J Ophthalmol 1998; 126:782-90.

11 Klein BEK, Klein R, Moss SE. Lens thickness and five-year cumulative incidence of cataracts. The Beaver Dam Eye Study. Ophthalmic Epidemiol 2000;7:243-8

12 Wong TY, Klein BEK, Klein R, et al. Refractive errors and incident cataracts: the Beaver Dam Eye Study. Invest Ophthalmol Vis Sci 2001;42:1449-54.

13 Adamsons I, Munoz B, Enger C, et al. Prevalence of lens opacities in surgical and general populations. Arch Ophthalmol 1991;109:993-7.

14 Congdon NC, Taylor HR. Age-related cataract. In: Johnson GJ, Minassian DC, Weale RA, West SK, eds. The epidemiology of eye disease. London: Arnold, 2003:106-7.

15 Congdon NG, Broman K, Lai H, et al. Nuclear cataract shows significant familial aggregation in an older population after adjustment for possible shared environmental factors. Invest Ophthalmol Vis Sci (in press).

16 Taylor HR, West SK. The clinical grading of lens opactities. Aust NZ J Ophthalmol 1989;17:81-6.

17 West SK, Rosenthal F, Newland HS, et al. Use of photographic techniques to grade nuclear cataracts. Invest Ophthalmol Vis Sci 1988;29:73-7.

18 StataCorp: Stata Statistical Software: Release 7.0. Stata Corporation, College Station, TX, 2000.

19 Eye Disease Prevalence Research Group. The prevalence of cataract and pseudophakia among adults in the United States. Arch Ophthalmol (in press).

20 Panchapakesan J, Mitchell $\mathrm{P}$, Tumuluri K, et al. Five year incidence of cataract surgery: the Blue Mountains Eye Study. Br J Ophthalmol 2003;87:168-72.

21 Jahn CE, Janke M, Winowski H, et al. Identification of metabolic risk factors for posterior subcapsular cataract. Ophthalmic Res 1986;18:112-6.

22 Wang JJ, Mitchell P, Simpson JM, et al. Visual impairment, age-related cataract, and mortality. Arch Ophthalmol 2001;119:1186-90.

23 West SK, Munoz B, Istre J, et al. Mixed lens opacities and subsequent mortality. Arch Ophthalmol 2000;1 18:393-7.

24 Borger $\mathrm{PH}$, van Leeuwen R, Hulsman CA, et al. Is there a direct association between age-related eye diseases and mortality? The Rotterdam Study. Ophthalmology 2003;110:1292-6. 